\title{
Radio activity in major mergings
}

\author{
E. Zucca ${ }^{1}$, T. Venturi ${ }^{2}$, S. Giacintucci ${ }^{1,3}$, S. Bardelli ${ }^{1}$ \\ and D. Dallacasa ${ }^{3}$ \\ ${ }^{1}$ INAF-Osservatorio Astronomico di Bologna, via Ranzani 1, 40127 Bologna, Italy e-mail: \\ elena.zucca@bo.astro.it \\ 2 INAF-Istituto di Radioastronomia, via Gobetti 101, 40129 Bologna, Italy \\ ${ }^{3}$ Dipartimento di Astronomia, Università di Bologna, via Ranzani 1, 40127 Bologna, Italy
}

\begin{abstract}
Cluster mergings are expected to have an influence on the radio emission of the galaxy population. We present the results of a deep radio survey in the A3558 complex in the central region of the Shapley Concentration, in order to further explore our hypothesis of a dependence of the radio luminosity function on the age of the merging.
\end{abstract}

\section{Introduction}

During the huge energy release of a cluster merging, different mechanisms are at work on galaxies. On one side, the rapid changes in the tidal gravitational field can drive gas from outer to inner parts of galaxies, triggering star bursts and feeding the central AGN (Bekki 1999), while on the other side tidal stripping and ram pressure tend to devoid galaxies of their hydrogen.

Owen et al. (1999) found that A2125, a merging cluster, presents a factor 4 more radio galaxies than a relaxed one (A2645) at the same distance. In order to better study this phenomenon, we performed an extensive radio survey at $20 \mathrm{~cm}$ with the ATCA telescope on the cluster complexes (Bardelli et al. 1998a, Bardelli et al. 1998b, Bardelli et al. 2000, Bardelli et al. 2002) located in the Shapley Concentration (Zucca et al. 1993).

The only way to estimate the radio activity of the clusters is to compute the bivariate radio-optical luminosity function (Figure 1), i.e. the number of radio galaxies in the considered structure (as confirmed by their redshift) over the total number of optical galaxies. This takes into account the different richness of the clusters. We found that the A3558 complex, which is a merging in an advanced phase, presents a significant lack of radiosources (Venturi et al. 2000, Venturi et al. 2001, Venturi et al. 2002). On the contrary the A3528 complex, which is at an early phase of interaction, presents a number of radiosources comparable to that of normal clusters. Also the A3571 complex, a merging at the end of the process, does not have pecularities in the number of radio objects.

This result is only apparently in contraddiction with Owen et al. (1999), because one has to take into account the spiral richness of interacting clusters and/or the merging phase. Our proposed scenario is the following: at very early stages of merging, the outer parts of clusters (which are spiral rich) begin to interact, triggering star formation activity and therefore enhancing the number of radiosources. After this episode, the radiosource excess disappears and the clusters return quiescent (right panel of Figure 1), until the ellipticals (with their AGNs) in the inner part of the clusters are reached by the merging effect, leading to a lowering of the AGN emission: as a result, there is a deficit of bright radiosources (left panel of Figure 1). Finally, the virialization re-establishes the starting situation. 

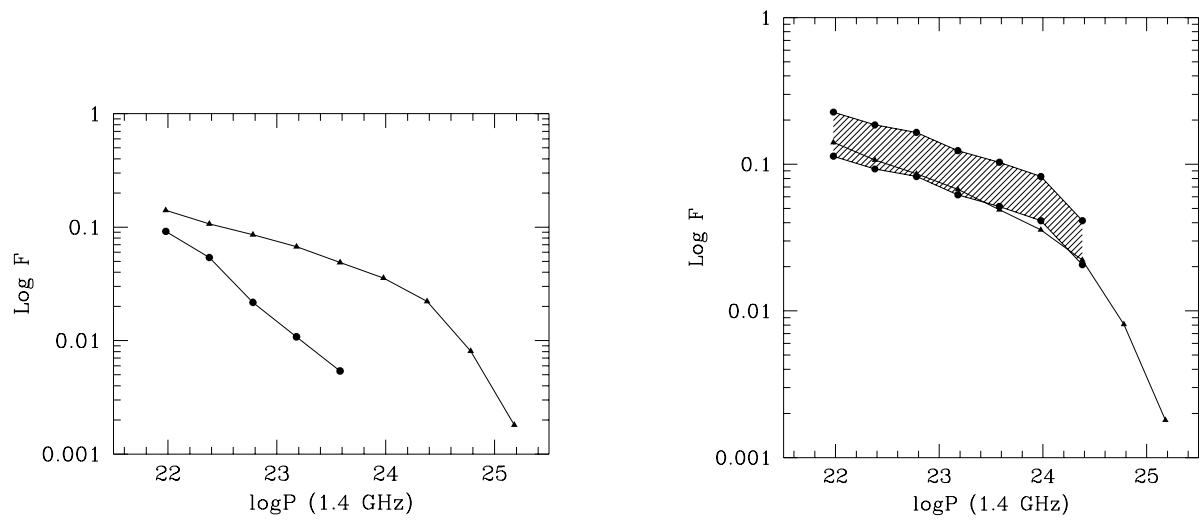

Figure 1. Bivariate radio-optical luminosity functions of the A3558 complex (left) and of the A3528 complex (right). Triangles represent the reference luminosity function of normal clusters from Ledlow \& Owen (1996). The first complex is an advanced merging, while the second is a merging at early phases. For the A3528 complex, the radio luminosity function lies in the shadowed area.
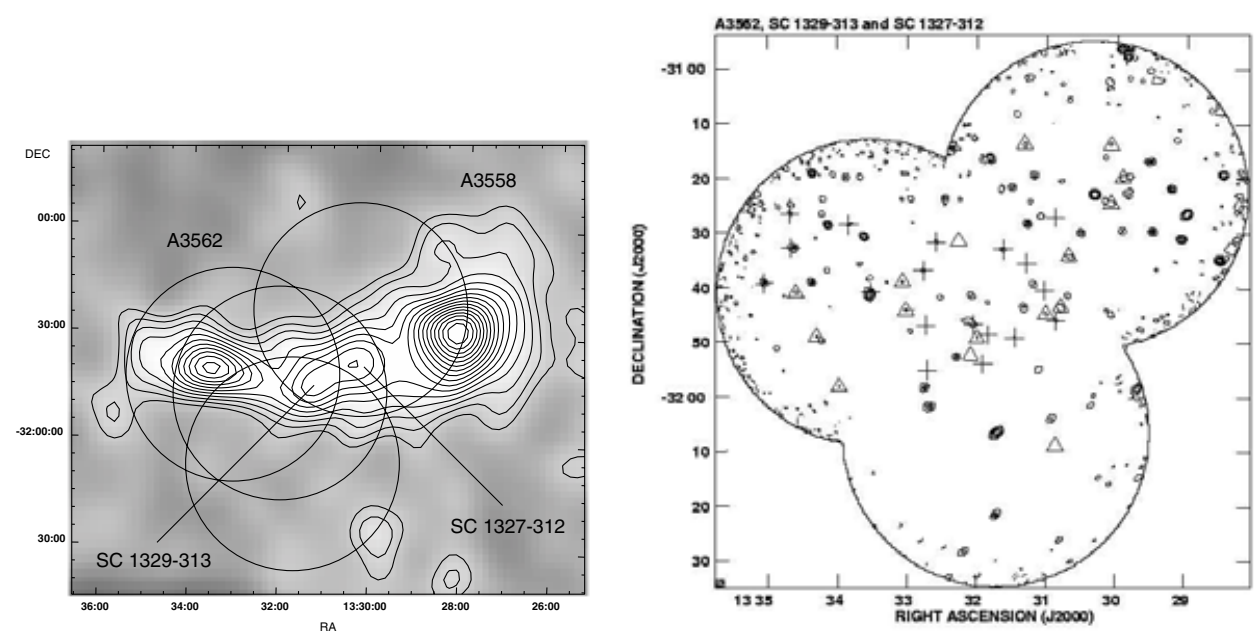

Figure 2. Left panel: circles represent the field of view of our VLA $20 \mathrm{~cm}$ pointings, superimposed to the isodensities of the optical distribution of galaxies in the A3558 complex. Right panel: location of optical counterparts overplotted on the radio contours. Triangles are late type galaxies, crosses are early type galaxies.

\section{The deep VLA survey}

In order to better study the lack of radiosources in the A3558 complex, we performed a dedicated, deep $\left(\sim 0.25 \mathrm{mJy}^{\text {beam }}{ }^{-1}\right)$ survey in the region between the two clusters A3558 and A3562 (see Figure 2, Giacintucci et al. 2004). This region is promising because it is highly substructured and presents features indicating an ongoing merging (see the contribution of Bardelli, this conference). Moreover, we discovered an interesting radio halo in the A3562 cluster (see the contribution of Giacintucci, this conference). The groups of this region tend to have bluer galaxies, indicating a possible ongoing starburst, and their hot gas distributions are highly disturbed (Bardelli et al. 2002). 
Given the high statistics, we computed the radio luminosity function for both early and late type galaxies in this region, finding consistency with Ledlow \& Owen (1996) for elliptical and S0 galaxies. Given the lack of bright radiosources that we found analysing the whole A3558 complex (see previous section), this result suggests that the deficit is entirely due to the A3558 cluster. Moreover, a population of faint $\left(\log P_{1.4 \mathrm{GHz}}\left[\mathrm{W} \mathrm{Hz}{ }^{-1}\right]<\right.$ 22) radio galaxies has been found: half of these objects are blue, suggesting that starburst is the driving radio emission mechanism. Finally, we found 14 spiral galaxies, whose ratio between radio and optical emission is similar to that of galaxies located in rich and dynamically evolved clusters.

The next steps of this analysis will be the following:

1) extending the study to other systems in other superclusters: in particular we are analysing the cluster A2061 in the Corona Borealis supercluster;

2) extending the survey to other wavelengths: in particular we obtained $610 \mathrm{MHz}$ observations of the A3558 complex with the GMRT telescope;

3) studying the HI emission of spiral galaxies in order to see whether the ongoing merging in the A3558 complex was able to strip gas from galaxies in between the dominant clusters.

\section{References}

Bardelli, S., Zucca, E., Zamorani, G., Vettolani, G., Scaramella, R. 1998a MNRAS 296, 599

Bardelli, S., Pisani, A., Ramella, M., Zucca, E., Zamorani, G. 1998b MNRAS 300, 589

Bardelli, S., Zucca, E., Zamorani, G., Moscardini, L., Scaramella, R. 2000 MNRAS 312, 540

Bardelli, S., De Grandi, S., Ettori, S., Molendi, S., Zucca, E., Colafrancesco, S. 2002 A 8 A 382, 17

Bekki, K. 1999 ApJ 510, L15

Giacintucci, S., Venturi, T., Bardelli, S., Dallacasa, D., Zucca, E. 2004 A\&A 419, 71

Ledlow, M.J. \& Owen, F.N. 1996 ApJ 112, 9

Owen, F.N., Ledlow, M.J., Keel, W.C., Morrison, G.E. 1999 AJ 118, 633

Venturi, T., Bardelli, S., Morganti, R., Hunstead, R.W. 2000 MNRAS 314, 594

Venturi, T., Bardelli, S., Zambelli, G., Morganti, R., Hunstead, R.W. 2001 MNRAS 324, 1131

Venturi, T., Bardelli, S., Zagaria, M., Prandoni, I., Morganti, R. 2002 A $\& A$ 385, 39

Zucca, E., Zamorani, G., Scaramella, R., Vettolani, G. 1993 ApJ 407, 470 